\title{
Montreal cognitive Assessment Score: A Screening Tool for Cognitive Function in Traumatic Brain Injury (TBI) Population
}

\author{
Keshav Mishra', Devendra Purohit ${ }^{1 *}$, Somnath Sharma' \\ 'Department of Neurosurgery, SMS Medical College, Jaipur, India
}

Article Info

\section{Article Notes}

Received: December 22, 2018

Accepted: August 11, 2020

\section{*Correspondence:}

Dr. Devendra Purohit, Senior Professor, Department of

Neurosurgery, SMS Medical college, Jaipur, India; Mobile No: +91 91982910335; E-mail: devendrapurohit@rediffmail.com.

() 2020 Purohit D. This article is distributed under the terms of the Creative Commons Attribution 4.0 International License

\begin{abstract}
Cognitive impairment is a major cause of morbidity and impaired quality of life in traumatic brain Injury (TBI) patients. Assessment of cognitive function using classically designed scales is time and resource intensive undertaking which also requires expert neuropsychiatrist referral. Montreal cognitive assessment Score (MoCA) is a brief screening tool designed to assess various cognitive domains which has been found to be more sensitive than Mini Mental State Examination (MMSE) score both in Alzheimer disease and subsequently in TBI population. Applied on TBI population, it reliably detects cognitive impairment in mild TBI, compared to normal controls and also differentiates cognitive disturbances between mild and severe TBI but its ability to differentiate cognitive function between mild and moderate TBI is equivocal.
\end{abstract}

\section{Introduction}

Traumatic Brain injury (TBI) is one of the most important global health issues which constitutes an increasingly common cause of mortality and a leading cause of morbidity in all age group, especially in young adults ${ }^{1}$. It affects the physical, psychosocial and cognitive skills in varying degrees thus causing significant deterioration in the quality of life and impairment of social and economic productivity ${ }^{2}$. With progressive evolution and introduction of modern monitoring systems and management strategies for TBI, patient survival has increased with concomitant increase in the number of TBI survivors battling with its long-term complications including cognitive dysfunction ${ }^{3}$.

The level of resultant cognitive impairment post TBI is seen to correlate with the severity of the brain injury ${ }^{4}$, with more grievous injuries resulting in severe and persistent cognitive dysfunction ${ }^{5}$. In a recent study, TBI has been seen to be associated with later development of dementia and Alzheimer disease ${ }^{6}$. The importance of early assessment of cognitive function post TBI lies in establishing a cognitive prognosis for patients and formulating early intervention plan. There is no single standard easily administrable tool to address these issues in TBI patients.

Montreal Cognitive Assessment (MoCA) score is a brief assessment tool of global cognitive function which was originally designed for early detection of mild cognitive deficits in patients with Alzheimer disease ${ }^{7}$ which has been subsequently validated in different cohorts. This screening tool uses various tasks to assess higher-level language abilities, memory and visuospatial and executive functions. MoCA is a freely available tool for use by medical professionals, clinics and hospitals involved in the care of such patients. It is available in over 30 languages. Various studies evaluating the presence of cognitive deficits 
at admission using MoCA have found patient`s age, severity of injury and educational status to be important factors affecting the patients score ${ }^{8}$.

\section{Discussion}

The cognitive outcome post TBI is affected by numerous injury related factors like severity of brain injury and its subsequent complications, associated injuries to other body regions, and the time scale of recovery from injury. Various patient related variables also play role in cognitive outcome such as patient's age, pre-injury neuropsychiatric and cognitive status as well as the quality of post discharge environment.

The Glasgow Coma Scale (GCS) score, duration of posttraumatic amnesia (PTA) and loss of consciousness (LOC) duration are the major factors used to analyze TBI severity. Using these characteristics, TBI can be classified: mild, moderate and severe. According to consensus definitions, moderate and severe TBI are characterized by loss of consciousness for greater than 30 minutes and/or PTA persisting for at least 24 hours while mild TBI (mTBI) may occur with or without loss of consciousness and PTA. TBI severity can also be defined according to the radiology findings at presentation using criteria such as Marshall classification ${ }^{9}$.

Executive functions, memory, visuospatial functions ${ }^{10}$, concentration disorders, deficits in information processing and speed ${ }^{11}$ are the most vulnerable cognitive functions affected by mild TBI. In general, 80-85 percent of patients with mTBI and with normal CT findings at admission show almost complete recovery of cognitive deficits ${ }^{12}$. But there is a sub-class in the mTBI group with who present with persistent cognitive impairment and the data delineating the etiology and further prognosis of this group of patients is unclear ${ }^{13}$. In a recent prospective study, it was seen that as many as one in every three mTBI patients continue to show some level of functional impairment upto three months post-injury ${ }^{14}$.

The spectrum of cognitive deficits in moderate and severe TBI is different than that seen in $\mathrm{mTBI}$, which tend to include awareness, reasoning, language, visuospatial processing, and general intelligence and they are seen to be more severe and persistent in nature ${ }^{15}$ with upto $65 \%$ of them reporting long-term cognitive dysfunction ${ }^{16}$.

The patients sustaining cognitive deficits post moderate to severe TBI show rapid improvement in the first year which is followed by the phase of gradual recovery subsequently with persistence of impairments in severely injured patients. Although recovery up to 5 years post injury has been documented in some patients, supposedly this is a function of adaptation and development of compensatory techniques over time ${ }^{17}$.
There is sufficient literature to show that neuropsychological test, as surrogate measures of cognitive ability, show positive and significant correlation with functional outcome measures (e.g. FIM and DRS score). In a study carried out by Hanks, neuropsychological assessment score was found to be a better predictor of 1-year outcome compared to functional outcome measures ${ }^{18}$.

The National Institutes of Health $(\mathrm{NIH})$, in order to ensure uniformity in collection and comparison of data obtained at different centers, has recommended a list of outcome measures referred to as Common Data Elements (CDEs) for clinical TBI research. The CDEs include tests for different aspects of cognitive assessment divided into core, basic, and supplemental categories ${ }^{19,20}$. Though a detailed analysis of cognition helps to better evaluate the baseline deficits, better plan the rehabilitation strategy as well as monitor its progress, it is a time intensive task which frequently requires referral to an expert neuropsychologist proficient in evaluation of cognitive function.

One of the tools developed recently to assess cognitive impairments following mTBI is MoCA score, devised in 2005 by Nasreddine et al. It was found to be more sensitive than MMSE score in differentiating early stages of cognitive decline $^{7}$. Since then its use has been extended to various patient cohorts like substance abuse disorders, Parkinson disease, geriatric patients, and post CVA patients. This test usually doesn't take more than $10 \mathrm{~min}$ to perform and with a maximum score of 30 points. The visuospatial/ executive category includes a clock-drawing task (3 points), a three-dimensional cube copy (1 point) and TrailMaking Test (1 point). Memory is assessed with a shortterm memory recall task ( 5 points) involving two learning trials of five nouns and delayed recall after approximately 5 min. Naming consists of a three-item confrontation task and the language assessment includes repetition of two syntactically complex sentences ( 2 points) and a phonemic fluency task (1 point). Attention is evaluated with a target detection task by tapping ( 1 point), a serial subtraction task (3 points), and digits forward and backward tasks (1 point each). A two-item verbal abstraction task ( 2 points) and finally the orientation to time and place is assessed ( 6 points) $)^{7}$.

A review comparing various brief assessment tools for cognitive function listed the MoCA's disadvantages as lack of sufficient literature validating and supporting its use in TBI patients, effect of education level on final score and administration time of over $10 \mathrm{~min}^{21}$. Nazem and colleagues in their study have shown male gender, increased age, lower literacy level and greater disease severity as predictive factors of obtaining lower scores on MoCA test ${ }^{22}$.

The Ontario Neurotrauma Foundation (ONF), in its 2013 edition has described best practices for TBI and suggested the use of MoCA score for screening for cognitive disorders ${ }^{23}$. 
In 2018 ONF in its updated guideline recommended the use of validated post-concussion questionnaire like Rivermead, PCSS or SCAT5 besides MoCA score for mTBI patients ${ }^{24}$. Despite the ONF recommendation, the literature investigating the utility of MoCA following TBI is sparse.

Wong et al in their study on 48 patients of traumatic ICH reported significant correlation of MoCA score with (mini mental state examination) MMSE and a standard battery of neurophysiological tests for assessment of cognitive impairment in traumatic ICH patients. Theyalso emphasized the utility of MoCA for prognostication and clinical follow up. Lim et al also demonstrated good alternate form testre-test stability of MoCA score with minimal practice effect between its different versions thus establishing it to be a reliable instrument for monitoring cognitive change during follow up of TBI patients ${ }^{25}$. However, in a comparative study by De guise et $\mathrm{al}^{26} \mathrm{MoCA}$ score was not found to be a better predictor of outcome than the MMSE for a TBI population in the acute care setting. This finding could be attributed to ceiling effect in the study population as these patients in the acute setting presented with maximum scores in the MMSE test.

Zhang and group assesed MoCA against a computerized battery of cognitive tests consisting of Stroop Test, the Symbol Digit Modalities Test (SDMT), and a visual version of the Paced Auditory Serial Addition Test 2-second trials (PVSAT-2) in 255 consecutive TBI patients. They reported superior sensitivity of computerized cognitive assessment compared to MoCA score with their computerized battery detecting impairment in 50\% subjects with normal MoCA score. They also highlighted the falloff of using single cut off score for MoCA rather than using a normative Z score cutoff adjusted for age and level of education in the given population. The limitation cited for this study were inability to stratify the sample according to TBI severity leading to a possibility of type II error and inability to control for potential confounding factors other than preinjury psychiatric status which could potentially influence the test outcome ${ }^{27}$.

In a retrospective study done by de Guise et al on 214 admitted TBI patients in a level I trauma centre, comparing patients with mild, moderate and severe TBI using the MoCA, it was found that Patients with mTBI and moderate TBI performed better on MoCA score than those with severe TBI. But no significant differences were observed between patients with mTBI and moderate TBI. Although when GCS score was analyzed as continuous data rather than groups, TBI severity did influence MoCA scores positively. This contrasting finding was attributed by the authors to the admission bias of more severely injured patient included in the mTBI group. It can also be argued that the lack of differentiation between mild and moderate TBI in this study could be due to lack of sensitivity of MoCA score to pick up the discriminating variables between the two groups or due to lack of discrimination in the severity classification using the GCS score. No correlation was observed between the severity of brain injury according to Marshall classification and extent of cognitive impairment using MoCA score. They also reported a significant difference between severe TBI and mTBI or moderate TBI group for the visuospatial/ executive, attention, and orientation cognitive domains, whereas no difference was observed in naming, language, abstraction, and memory. Age, education status and presence of preexisting neurological problems were also significantly related to MoCA score.

As some of the MoCA test items are dependent on the age and education status of the test subjects so it supposedly skews the score in favor of younger population with more years of education. Chen et al introduced a modification of the MoCA score, Montreal Cognitive Assessment-Basic (MoCA-B) scale for use in subjects with lower literacy levels ${ }^{28}$. An et al further explored the utility of the scale in the TBI population ${ }^{29}$. They found age and educational status to be a negative and positive correlate respectively with the MoCA-B scores. They also reported higher test scores for patients with higher GCS. The site of lesion and gender were not found to have significant correlation with test score. However, Panwar et al in their prospective study on 228 patients, reported lesion location and type to be a predictor of test performance ${ }^{30}$. They found more bilateral and diffuse lesion in moderate groups compared to unilateral frontal lesions in mild group. GCS as a linear score was also found to be associated with MoCA score with more severe injury having more cognitive impairments particularly in executive function, attention, concentration and memory domains ${ }^{30}$.

Frenette and collegues ${ }^{31}$ studied the utility of MoCA in 42 uncomplicated TBI, 92 complicated TBI and 50 healthy controls. They showed significant differences between healthy control participants and patients with mTBI on the MoCA total score and more specifically in the visuospatial/ executive and language subtests. But this study failed to show significant differences between uncomplicated and complicated (cerebral lesions on imaging) mTBI groups. The literature review also outlined similar discrepancies where some studies ${ }^{32-34}$ have shown increased cognitive impairment in complicated TBI compared to uncomplicated TBI. However, Carroll ${ }^{35}$ and de Guise ${ }^{36}$ in their study didn't find any significant difference in neurophysiological evaluation in uncomplicated and complicated TBI patients in acute setting. The study showed association between performance on naming task to be associated to presence of frontal and temporal lesions. This study also failed to show clear relationship between GCS and MoCA scores though performances on the naming, attention, abstraction, and orientation subtests were associated with the GCS scores. 


\section{Conclusion}

MoCA is a brief and easily administrable test for global assessment of cognitive function which has proved useful to screen for presence of cognitive impairment in the outpatient setting by trained healthcare personal. Though it cannot replace the comprehensive battery of neurophysiological assessment for cognitive impairment and its usefulness in differentiating the cognitive impairment between complicated and uncomplicated mTBI is debatable, it reliably detects impairment in mild TBI and differentiates cognitive disabilities between mild to severe TBI.

\section{References}

1. Plata CDM, Hart T, Hammond FM, et al. Impact of age on long-term recovery from traumatic brain injury. Arch Phys Med Rehabil. 2008; 89: 896-903.

2. Mackenzie A, Alfred D, Fountain R, et al. Quality of life and adaptation for traumatic brain injury survivors: Assessment of the disability centrality model. J Rehab. 2015; 81(3): 9.

3. Bigler E. Neuropsychology and clinical neuroscience of persistent $p$ cussive syndrome. J IntNeuropsycholSoc. 2008; 14: 1-22.

4. Draper K, Ponsford J. Cognitive functioning ten years following traumatic brain injury and rehabilitation. Neuropsy. 2008; 22(5): 618.

5. Rabinowitz AR, Levin HS. Cognitive sequelae of traumatic brain injury. PsychiatrClin North Am. 2014; 37: 1-11.

6. Sivanandam TM, Thakur MK. Traumatic brain injury: a risk factor for Alzheimer's disease. Neurosci Biobehav Rev. 2012; 36: 1376-81.

7. Nasreddine ZS, Phillips NA, Bédirian V, et al. The Montreal Cognitive Assessment, MoCA: a brief screening tool for mild cognitive impairment. Journal of the American Geriatrics Society. 2005 Apr; 53(4): 695-9.

8. De Guise E, Alturki AY, LeBlanc J, et al. The Montreal Cognitive Assessment in persons with traumatic brain injury. Applied Neuropsychology: Adult. 2014 Apr 1; 21(2): 128-35.

9. Marshall LF, Marshall SB, \&Klauber MR. A new classification of head injury based on computerized tomography. Journal of Neurosurgery. 1991; 75(Suppl 1): S14-S20.

10. Karr JE, Areshenkoff CN, Garcia-Barrera MA. The neuropsychological outcomes of concussion: a systematic review of meta-analyses on the cognitive sequelae of mild traumatic brain injury. Neurology. 2014; 28: 321-36.

11. Carroll LJ, Cassidy JD, Cancelliere C, et al. Systematic review of the prognosis after mild traumatic brain injury in adults: cognitive, psychiatric, and mortality outcomes: results of the international collaboration on mild traumatic brain injury prognosis. Arch Phys Med Rehabil. 2014; 95: S152-S73. doi:10.1016/j.apmr.2013.08.300.

12. Belanger HG, Vanderploeg RD. The neuropsychological impact of sports-related concussion: a meta-analysis. Journal of the International Neuropsychological Society. 2005; 11(4): 345-57. [PubMed: 16209414]

13. Roe C, Sveen U, Alvsaker K, et al. Post-concussion symptoms after mild traumatic brain injury: influence of demographic factors and injury severity in a 1-year cohort study. Disability \& Rehabilitation. 2009; 31(15): 1235-43. [PubMed: 19116810]

14. McMahon P, Hricik A, Yue JK, et al. Symptomatology and Functional Outcome in Mild Traumatic Brain Injury: Results from the Prospective TRACK-TBI Study. Journal of Neurotrauma. 2013.
15. Pagulayan KF, Temkin NR, Machamer JE, et al. The measurement and magnitude of awareness difficulties after traumatic brain injury: a longitudinal study. Journal of the International Neuropsychological Society. 2007; 13(4): 561-70.

16. Whiteneck GG, Gerhart KA, Cusick CP. Identifying environmental factors that influence the outcomes of people with traumatic brain injury. The Journal of Head Trauma Rehabilitation. 2004; 19(3): 191204.

17. Corrigan JD. Consequences of traumatic brain injury for functioning in the community. Rehabilitation of Persons With Traumatic Brain Injury. 1998; 51.

18. Hanks RA, Millis SR, Ricker JH, et al. The predictive validity of a brief inpatient neuropsychologic battery for persons with traumatic brain injury. Arch Phys Med Rehabil. 2008; 89: 950-7.

19. Wilde EA, Whiteneck GG, Bogner J, et al. Recommendations for the use of common outcome measures in traumatic brain injury research. Arch Phys Med Rehabil. 2010; 91(11): 1650-60. [PubMed: 21044708]

20. Hicks R, Giacino J, Harrison-Felix C, et al. Progress in developing common data elements for traumatic brain injury research: version two - the end of the beginning. Journal of Neurotrauma. 2013; 30(22): 1852-61.

21. Cordell C, Borson B, Boustani M, et al. Alzheimer's Association recommendations for operationalizing the detection of cognitive impairment during the Medicare Annual Wellness Visit in a primary care setting. Alzheimers Dement. 2013; 9: 141-150.

22. Nazem S, Siderowf AD, Duda JE, et al. Montreal Cognitive Assessment performance in patients with Parkinson's disease with 'normal' global cognition according to Mini-Mental State Examination score. Journal of the American Geriatrics Society. 2009; 57: 304-308.

23. Ontario NeurotraumatologyFondation. Guidelines for concussion mild traumatic brain injury \& persistent symptoms. 2nd ed. [Internet]. Toronto (ON); 2013 Sept. http://onf.org/system/attachments/222/ original/ONF_mTBI_Guidelines_2nd_Edition_CLINICAL.pdf.

24. Ontario Neurotrauma Foundation. Guidelines for concussion/mTBI \& persistent symptoms. $3^{\text {rd }}$ ed. [internet]. http://braininjuryguidelines. org/concussion/fileadmin/media/adult-concussion-guidelines-3rdedition.pdf

25. Lim PA, McLean AM, Kilpatrick C, et al. Temporal stability and responsiveness of the Montreal Cognitive Assessment following acquired brain injury. Brain injury. 2016 Jan 2; 30(1): 29-35.

26. de Guise E, LeBlanc J, Champoux MC, et al. The mini-mental state examination and the Montreal Cognitive Assessment after traumatic brain injury: an early predictive study. Brain injury. 2013 Nov 1; 27(12): 1428-34.

27. Zhang JY, Feinstein A. Screening for cognitive impairments after traumatic brain injury: A comparison of a brief computerized battery with the Montreal cognitive assessment. The Journal of neuropsychiatry and clinical neurosciences. 2016 0ct; 28(4): 328-31.

28. Chen KL, Xu Y, Chu AQ, et al. Validation of the Chinese Version of Montreal Cognitive Assessment Basic for Screening Mild Cognitive Impairment. J Am Geriatr Soc. 2016; 64(12): e285-e290. doi:10.1111/ jgs. 14530

29. Jing An, Qilong Cao, Wei Lin, et al. Cognition in patients with traumatic brain injury measured by the Montreal Cognitive Assessment-Basic, Applied Neuropsychology: Adult. 2019.

30. Panwar N, Purohit D, Deo Sinha V, et al. Evaluation of extent and pattern of neurocognitive functions in mild and moderate traumatic brain injury patients by using Montreal cognitive assessment (MoCA) score as a screening tool: An Observational study from India, Asian Journal of Psychiatry. 2018. https://doi.org/10.1016/j.ajp.2018.08.007 
31. Frenette LC, Tinawi S, Correa JA, et al. Early detection of cognitive impairments with the Montreal Cognitive Assessment in patients with uncomplicated and complicated mild traumatic brain injury. Brain injury. 2018 Nov 3; 1-9.

32. Iverson GL, Franzen MD, Lovell MR. Normative comparisons for the controlled oral word association test following acute traumatic brain injury. ClinNeuropsychol. 1999; 13: 437-41. doi:10.1076/ 13854046(199911)13:04;1-Y;FT437.

33. Lange RT, Iverson GL, Zakrzewski MJ, et al. Interpreting the trail making test following traumatic brain injury: comparison of traditional time scores and derived indices. J ClinExpNeuropsychol. 2005; 27: 897-06. doi:10.1080/1380339049091290.
34. Iverson G. Complicated vs uncomplicated mild traumatic brain injury: acute neuropsychological outcome. Brain Inj. 2006; 20: 1335-44. doi:10.1080/02699050601082156.

35. Carroll LJ, Cassidy JD, Cancelliere C, et al. Systematic review of the prognosis after mild traumatic brain injury in adults: cognitive, psychiatric, and mortality outcomes: results of the international collaboration on mild traumatic brain injury prognosis. Arch Phys Med Rehabil. 2014; 95: S152-S73. doi:10.1016/j.apmr.2013.08.300

36. de Guise E, Lepage JF, Tinawi S, et al. Comprehensive clinical picture of patients with complicated vs uncomplicated mild traumatic brain injury. ClinNeuropsychol. 2010; 24: 1113-30. doi:10.1080/1385404 6.2010 .506199 . 